\title{
Model-Matching-Based Control of the Beverton-Holt Equation in Ecology
}

\author{
M. De La Sen and S. Alonso-Quesada \\ Department of Electricity and Electronics, Faculty of Science and Technology, \\ Institute of Research and Development of Processes, Campus of Leioa, 48940 Leioa Bilbao, Spain
}

Correspondence should be addressed to M. De La Sen, manuel.delasen@ehu.es

Received 24 July 2007; Revised 24 September 2007; Accepted 16 November 2007

Recommended by Juan Jose Nieto

\begin{abstract}
This paper discusses the generation of a carrying capacity of the environment so that the famous Beverton-Holt equation of Ecology has a prescribed solution. The way used to achieve the tracking objective is the design of a carrying capacity through a feedback law so that the prescribed reference sequence, which defines the suitable behavior, is achieved. The advantage that the inverse of the Beverton-Holt equation is a linear time-varying discrete dynamic system whose external input is the inverse of the environment carrying capacity is taken in mind. In the case when the intrinsic growth rate is not perfectly known, an adaptive law implying parametrical estimation is incorporated to the scheme so that the tracking property of the reference sequence becomes an asymptotic objective in the absence of additive disturbances. The main advantage of the proposal is that the population evolution might behave as a prescribed one either for all time or asymptotically, which defines the desired population evolution. The technique might be of interest in some industrial exploitation problems like, for instance, in aquaculture management.
\end{abstract}

Copyright ( 2008 M. De La Sen and S. Alonso-Quesada. This is an open access article distributed under the Creative Commons Attribution License, which permits unrestricted use, distribution, and reproduction in any medium, provided the original work is properly cited.

\section{Introduction}

The nonautonomous discrete so-called Beverton-Holt equation (BHE) is very common in Ecology and, in particular, in studying the growth population dynamics (see, e.g., [1-9]). The equation is of great importance in the fishery industry concerning the growth and exploitation of species like, for instance, plaice, haddock and coho salmon, as well as other bottom feeding fish populations [1]. It is proven that, for subcritical biomass, according to a low value of the intrinsic growth rate of the studied population, zero is a stable attractor if the carrying capacity is not independent of the intrinsic growth rate in order that the model is well posed. In particular, the carrying capacity should decrease as the spawning stock decreases converging to zero. The BHE is a two-parameter widely used nonlinear equation of the form [1-9] 


$$
x_{k+1}=\frac{\mu_{k} K_{k} x_{k}}{K_{k}+\left(\mu_{k}-1\right) x_{k}}, \quad k \in \mathbf{N}_{0}:=\mathbf{N} \cup\{0\},
$$

with $\mathbf{N}$ being the set of natural numbers, where $x_{0}>0, \mu_{k} \in \mathbf{R}_{0}^{+}:=\mathbf{R}^{+} \cup\{0\}\left(\mathbf{R}^{+}\right.$being the set of positive real numbers) is the intrinsic growth rate of the population, determined by life cycle and demographic properties (specie growth rate, survivorship rate, etc.), and $K_{k} \in \mathbf{R}^{+}$, so-called the environment carrying capacity, is a characteristic of the habitat being dependent on resources availability, temperature, humidity, and so on. Typically, $\mu_{k}>1$ and $K_{k}=K_{k+p} \in \mathbf{R}_{0}^{+}$, for some $p(\geq 2) \in \mathbf{N}$, due to periodic fluctuations. It has been reported that a negative carrying capacity is nonsense since a small carrying capacity is sufficient to interpret a very unfavorable situation for the population [6], even to model the so-called Allee effect, that is, the difficulty of finding mates due to the very scarce number of population [7]. Note that $K_{k}=0$ is sufficiently unfavorable to extinguish the population since, from (1.1), a recruitment $x_{k+1}=0$ would occur for any spawning stock. The population extinction has been dealt with in [10] for intrinsic growth rates less than unity by discussing the spawning stock biomass conditions for the zero equilibrium to be a stable attractor. It has been concluded that the environment carrying capacity is not independent of the intrinsic growth rate for subcritical spawning stock biomass. Generalizations of the BHE including bounded discontinuities at sampling points, which can be interpreted as the consequence of impulsive effects [11], in the generating continuous logistic equation, have been also studied.

This paper is devoted to the model matching of a prescribed reference model which is also defined in practice by a BHE. The standard case of intrinsic growth-rate sequences being greater than unity is considered. The environment carrying capacity is locally modified around its reference values to achieve the prescribed behavior. Its inverse plays the role of the control of the IBHE, namely, the inverse of the BHE which is a linear dynamic system [9], and then much easier to deal with than the BHE. The control law consists of calculating the appropriate carrying capacity inverse so that the solution of the IBHE (namely, the inverse of the population levels) coincides with the solution of the reference IBHE. In that way, the population levels also coincide with the reference-suited ones. In the case when the intrinsic growth rate is not exactly known, the scheme is extended by incorporating parametrical estimation and a related adaptive control law. The tracking objective becomes asymptotic as a result of the incorporation of the estimation process. Since, in many practical situations, the reference model and the current one are locally deviated from each other, the solution matches very closely the reference solution for all time. The proposed technique is very feasible in close or semi-open environments like, for instance, aquaculture industry, for instance, by local modification of the temperature. In the case when the BHE parameters are not exactly known, an adaptive extension of the method incorporating parameter estimation is used instead. The paper is organized as follows. Section 2 is devoted to the statement of a control law for the case of known parameters. The BHE is generalized to include, if suited, additive disturbances which may be useful to describe uncertainties in the parameters of the BHE or phenomena which are not included in the nominal standard equation like, for instance, local or global migrations of the population. The reference model to be tracked (also often referred to as "matched" in the literature) is also a BHE which potentially includes additive disturbances corresponding to those present in the given BHE being defined as that possessing as its solution, the IBHE of the given BHE. Such an inverse has a linear structure by nature so that it is easy to deal with, and it describes the appropriate system behavior. The control law is of a feedback type and consists of designing appropriately the environment carrying capacity, within some prescribed margins, so that the 
reference model is perfectly tracked (i.e., the solution of the controlled equation and that of the reference model coincide for all samples). This is very feasible in closed environments like, for instance, fisheries. It turns out that the achievement of a perfect tracking of the IBHE corresponds to achieving the objective of a perfect tracking of the corresponding reference BHE. Section 3 extends the method to the adaptive case by incorporating an adaptive scheme. It is assumed that certain a priori knowledge on the additive disturbances is available so that a relative dead zone is used in the adaptation algorithm to freeze the adaptation under smalladaptation errors to prevent against stability caused by the disturbances when the parameters are not fully known [12]. The adaptive control law is implemented by replacing the controller parameters in the case of perfect knowledge by those computed based on the estimates of the IBHE. The closed-loop system is proven to be stable if the additive disturbances grow, at most linearly, with the maximum of the solution of the IBHE. In the adaptive case, the tracking objective is only achievable asymptotically provided that the contribution of the additive disturbances is zero or becomes extinguished asymptotically. The method to discuss the stability and convergence of estimates relies on the use of a Lyapunov sequence, $[8,13,14]$. Section 4 presents two numerical examples and, finally, conclusions end the paper.

\section{Control law for the case of known parameters}

Define an inverse system of (1.1) through the change of variable $s_{k}=x_{k}^{-1}$ resulting directly from (1.1) in the time-varying discrete linear system

$$
s_{k+1}=a_{k} s_{k}+b_{k} u_{k}, \quad s_{0}=x_{0}^{-1}>0,
$$

where $a_{k}=\mu_{k}^{-1}, b_{k}=1-\mu_{k}^{-1}=1-a_{k}$, and $u_{k}=K_{k}^{-1}$, that is, the inverse carrying capacity is the control action. If there exists an additive disturbance sequence $\eta_{k}$ in (2.1), one gets, instead of (2.1) [9],

$$
s_{k+1}=a_{k} s_{k}+b_{k} u_{k}+\eta_{k}
$$

The IBHE (2.2) corresponds, through the change of variable $x_{k}=s_{k}^{-1}$, to an extended BHE which includes a sequence of additive disturbances:

$$
\begin{aligned}
x_{k+1} & =\frac{K_{k} x_{k}}{a_{k} K_{k}+\left(b_{k}+K_{k} \eta_{k}\right) x_{k}}=\frac{\mu_{k} K_{k} x_{k}}{K_{k}+\left(\mu_{k}-1+\mu_{k} K_{k} \eta_{k}\right) x_{k}} \\
& =\frac{\mu_{k} K_{k} x_{k}}{K_{k}+\left(\mu_{k}-1\right) x_{k}+v_{k}^{0}}=\frac{\mu_{k} K_{k} x_{k}}{K_{k}+\left(\mu_{k}-1\right) x_{k}}+v_{k},
\end{aligned}
$$

where

$$
\begin{aligned}
v_{k}^{0} & =\mu_{k} K_{k} \eta_{k} x_{k}, \\
v_{k} & =-\frac{\mu_{k} K_{k} x_{k} v_{k}^{0}}{\left(K_{k}+\left(\mu_{k}-1\right) x_{k}\right)\left(K_{k}+\left(\mu_{k}-1+\mu_{k} K_{k} \eta_{k}\right) x_{k}\right)} \\
& =-\frac{\mu_{k}^{2} K_{k}^{2} x_{k}^{2} \eta_{k}}{\left(K_{k}+\left(\mu_{k}-1\right) x_{k}\right)\left(K_{k}+\left(\mu_{k}-1+\mu_{k} K_{k} \eta_{k}\right) x_{k}\right)} .
\end{aligned}
$$


Note that the sequence $\left\{v_{k}\right\}_{0}^{\infty}$ is an additive disturbance of the BHE which is identically zero if the additive disturbance sequence $\left\{\eta_{k}\right\}_{0}^{\infty}$ of its inverse is identically zero. The disturbance may include the effects in the solution of parametrical uncertainties, for instance, in the intrinsic growth rate, or effects like, for instance, migrations or local migrations which are not taken into account in the standard BHE. The following assumptions are concerned with the stability and controllability of the IBHE and with the knowledge of an upper bound of the disturbance term if such a disturbance is unknown.

Assumption 2.1. $\infty>\mu_{k} \geq 1+\varepsilon_{\mu}$ and $\infty>K_{k} \geq \varepsilon_{K}$, for all $k \in \mathbf{N}_{0}$, and some $\varepsilon_{\mu} \in \mathbf{R}^{+}, \varepsilon_{K} \in \mathbf{R}^{+}$.

Assumption 2.2. $\eta_{k}\left(\in \mathbf{R}_{0}^{+}\right) \leq \bar{\eta}_{k}$, for all $k \in \mathbf{N}_{0}$, and $\left\{\bar{\eta}_{k}\right\}_{0}^{\infty}$ is known.

Note that Assumption 2.1 implies that the BHE is stable since $\mu_{k}^{-1}<1$, and also controllable since $b_{k} \geq \varepsilon_{\mu}^{\prime}:=\varepsilon_{\mu} /\left(1+\varepsilon_{\mu}\right)>0$ so that $\left(1-\mu_{k}^{-1}\right)$, for all $k \in \mathbf{N}_{0}$, so that $s_{k}$ in (2.2), and then the BHE solution $x_{k}=s_{k}^{-1}$ may be driven to sequences of prescribed values. These two properties are crucial for the solution to track a prescribed reference sequence. Note also that $\varepsilon_{\mu}$ may be chosen in the real interval $(0,1)$ without any loss in generality since, if Assumption 2.1 holds for any given $\varepsilon_{\mu}>0$, then it always holds for some $\varepsilon_{\mu} \in(0,1)$. This fact is used in the proof of the subsequent result, which is direct and related to the positivity and boundedness of the solution.

Assertion 2.3. If Assumptions 2.1 and 2.2 hold and $\left\{\eta_{k}\right\}_{0}^{\infty}$ is uniformly bounded, then all solutions of the BHE and the IBHE are uniformly bounded and positive, provided that $\infty>x_{0}>0$.

Proof. $\mu_{k} \geq 1+\varepsilon_{\mu} \Leftrightarrow b_{k} \in\left[\varepsilon_{\mu}^{\prime}, 1-\varepsilon_{\mu}^{\prime}\right]$. Define $\rho:=\operatorname{Max}_{0 \leq k<\infty}\left(\mu_{k}^{-1}\right) \in\left(0,\left(1 /\left(1+\varepsilon_{\mu}^{\prime}\right)\right)\right] \subseteq(0,1)$. Since $s_{0}=x_{0}^{-1}>0$, it follows from (2.2) that

$$
\begin{aligned}
0<x_{k}^{-1} & =s_{k} \leq \rho^{k} s_{0}+\sum_{i=0}^{k-1} \rho^{k-i-1}\left(1-\varepsilon_{\mu}^{\prime}\right)\left(K_{i}^{-1}+\eta_{i}\right) \\
& \leq \rho^{k} s_{0}+\frac{1-\rho^{k}}{1-\rho}\left(1-\varepsilon_{\mu}^{\prime}\right) \operatorname{Max}_{0 \leq i<\infty}\left(K_{i}^{-1}+\eta_{i}\right) \leq s_{0}+\frac{\left(1-\varepsilon_{\mu}^{\prime 2}\right)}{\varepsilon_{\mu}^{\prime} \varepsilon_{K}} \operatorname{Max}_{0 \leq i<\infty}\left(\eta_{i}\right)<\infty \\
& \Longleftrightarrow x_{k} \in(0, \infty), \quad \forall k \in \mathbf{N}_{0}, \text { since } \frac{1}{1-\rho} \leq \frac{1-\varepsilon_{\mu}^{\prime}}{\varepsilon_{\mu}^{\prime}}, \operatorname{Max}_{0 \leq i<\infty}\left(K_{i}^{-1}\right) \leq \frac{1}{\varepsilon_{K}} .
\end{aligned}
$$

The subsequent set of structures is considered in order to be then able to formulate the control law.

\section{(1) A reference}

BHE $x_{k+1}^{*}=\mu_{k}^{*} K_{k}^{*} x_{k}^{*} /\left(K_{k}^{*}+\left(\mu_{k}^{*}-1\right) x_{k}^{*}\right)$, for all $k \in \mathbf{N}_{0}$, which defines the suitable solution through the appropriate reference values of the intrinsic growth rate and the environment carrying capacity of its corresponding reference IBHE $s_{k+1}^{*}=a_{k}^{*} s_{k}^{*}+b_{k}^{*} r_{k}$, with reference input $r_{k}=\left(K_{k}^{*}\right)^{-1}>0$, and parameter sequences $a_{k}^{*}=\left(\mu_{k}^{*}\right)^{-1}<1$ and $b_{k}^{*}=\left(\mu_{k}^{*}\right)^{-1}>0$. 
(2) The current

BHE $x_{k+1}=\mu_{k} K_{k} X_{k} /\left(K_{k}+\left(\mu_{k}-1\right) x_{k}\right)+v_{k}$ and its corresponding IBHE

$$
s_{k+1}=a_{k} s_{k}+b_{k} u_{k}+\eta_{k}
$$

whose parameters are $a_{k}=\mu_{k}^{-1}, b_{k}=1-\mu_{k}^{-1}$ are deviated from the suited ones $a_{k}^{*}=\left(\mu_{k}^{*}\right)^{-1}$ and $b_{k}^{*}=1-\left(\mu_{k}^{*}\right)^{-1}$, which may include disturbance terms grouped in a sequence $\left\{v_{k}\right\}_{0}^{\infty}$ with control input $u_{k}=K_{k}^{-1}$ obtained according to a feedback law.

(3) The control law:

$$
u_{k}=t_{k} r_{k}+f_{k} s_{k}-\omega_{k}
$$

provided that $\eta_{k}$ is known, which is a relaxation of Assumption 2.2 which is parameterized by

$$
f_{k}=\frac{a_{k}^{*}-a_{k}}{b_{k}}, \quad t_{k}=\frac{b_{k}^{*}}{b_{k}}, \quad \omega_{k}=\frac{\eta_{k}}{b_{k}}
$$

with $b_{k}=1-a_{k}=1-\mu_{k}^{-1}$. Note that the control law generates the inverse carrying capacity from the reference inverse carrying capacity and the solution of the IBHE with two parametrical sequences $\left\{f_{k}\right\}_{0}^{\infty}$ and $\left\{t_{k}\right\}_{0}^{\infty}$ and a correcting sequence $\left\{\omega_{k}\right\}_{0}^{\infty}$ calculated from (2.8). The subsequent constraint

$$
r_{k}=\left(K_{k}^{*}\right)^{-1}>\frac{\omega_{k} x_{k}-f_{k}}{t_{k} x_{k}}=\frac{1}{t_{k}}\left(\frac{\eta_{k}}{1-a_{k}}+\frac{a_{k}-a_{k}^{*}}{x_{k}}\right)
$$

must hold for all samples to guarantee that the environment carrying capacity is positive. The following result establishes that the tracking error in between the reference sequence and the current solution for the given control law is zero. In that way, the perfect tracking objective is achieved if the disturbance sequence is known.

Proposition 2.4. If $s_{0}=s_{0}^{*}$, then the control law (2.7)-(2.8) achieves an identically zero tracking error $\left\{\varepsilon_{k}:=s_{k}-s_{k}^{*}\right\}_{0}^{\infty}$.

Proof. It follows, by complete induction, by assuming $s_{k}=s_{k}^{*} \Leftrightarrow \varepsilon_{k}=0$, for all $k \in \mathbf{N}_{0}$, that

$$
\varepsilon_{k+1}=\left(a_{k}+b_{k} f_{k}-a_{k}^{*}\right) s_{k}^{*}+\left(b_{k} t_{k}-b_{k}^{*}\right) r_{k}=0, \quad \forall k \in \mathbf{N}_{0} .
$$

If the disturbance is unknown, but Assumption 2.2 still holds, then the last identity in the parameterization of the control law in (2.8) is replaced, with similar expressions, with the disturbances absolute upper bounds, namely, $\omega_{k}=\bar{\eta}_{k} / b_{k}$, where $\left|\eta_{k}\right| \leq \bar{\eta}_{k}$. Thus the tracking error is not identically zero but it is uniformly bounded. It is also very close to zero, provided that the deviation of the upper bound of the disturbance with respect to such a disturbance is very close to zero. Then one has the subsequent result. 
Proposition 2.5. If Assumption 2.1 holds and $\left|\eta_{k}\right| \leq \bar{\eta}_{k}$ with $\eta_{k}$ being unknown, but $\bar{\eta}_{k}$ being known, then the control law (2.7)-(2.8) of Proposition 2.4, with the modification $\omega_{k}=\bar{\eta}_{k} / b_{k}$, achieves a uniformly bounded tracking error $\left\{\varepsilon_{k}:=s_{k}-s_{k}^{*}\right\}_{0}^{\infty}$, provided that the disturbance sequence is upper bounded by a bounded sequence.

Proof. Direct calculations yield

$$
s_{k+1}=a_{k} s_{k}+b_{k} u_{k}+\eta_{k}, \quad s_{k+1}^{*}=a_{k}^{*} s_{k}^{*}+b_{k}^{*} r_{k}
$$

so that, from (2.7)-(2.8),

$$
\varepsilon_{k+1}=a_{k}^{*} \varepsilon_{k}+\left(\eta_{k}-\bar{\eta}_{k}\right) \leq\left(a^{*}\right)^{k+1} \varepsilon_{0}+\frac{m_{\eta}}{1-a^{*}}<\infty, \quad \forall k \in \mathbf{N}_{0},
$$

where $0<a_{k}^{*} \leq a^{*}<1$, for all $k \in \mathbf{N}_{0}$, and

$$
m_{\eta}:=\underset{0 \leq i<\infty}{\operatorname{Max}}\left(\left|\bar{\eta}_{i}-\eta_{i}\right|\right) \leq \underset{0 \leq i<\infty}{2 \operatorname{Max}}\left(\bar{\eta}_{i}\right)<\infty
$$

Since the inverse of the environment carrying capacity is the control action, a large deviation from its nominal values for tracking purposes may be not admissible. Note that Assumption 2.1 also applies to the reference to be tracked by the control action consisting of appropriate achievement of an environment carrying capacity, that is, $\infty>\mu_{k}^{*} \geq 1+\varepsilon_{\mu}>1$, and $\infty>K_{k}^{*} \geq \varepsilon_{k}>0$, for all $k \in \mathbf{N}_{0}$. That means that the maximum deviation of the suited reference sequence, related to the current solution, depends on the maximum allowed variations of the environment carrying capacity with respect to its nominal value. More precisely, the subsequent result follows.

Proposition 2.6. Assume that the maximum allowed absolute variation of the carrying capacity with respect to its nominal value is $\Delta_{K}>0$. Then the following properties hold.

(i) The control parameter sequence $\left\{t_{k}\right\}_{0}^{\infty}$ is subject to the constraint $t_{k} \in\left[1-\Delta_{K} / K_{k^{\prime}}^{*} 1+\Delta_{K} / K_{k}^{*}\right]$, for all $k \in \mathbf{N}_{0}$, which induces the parametrical constraints

$$
a_{k} \in\left[\frac{K_{k}^{*} a_{k}^{*}-\Delta_{K}}{K_{k}^{*}-\Delta_{K}}, \frac{K_{k}^{*} a_{k}^{*}+\Delta_{K}}{K_{k}^{*}+\Delta_{K}}\right], \quad b_{k} \in\left[\frac{K_{k}^{*} b_{k}^{*}}{K_{k}^{*}+\Delta_{K}}, \frac{K_{k}^{*} b_{k}^{*}}{K_{k}^{*}-\Delta_{K}}\right] .
$$

(ii) The tracking-error sequence $\left\{\varepsilon_{k}:=s_{k}-s_{k}^{*}\right\}_{0}^{\infty}$ is subject to the constraint

$$
\left(\varepsilon_{k+1}-a_{k} \varepsilon_{k}+\left(a_{k}^{*}-a_{k}\right) s_{k}^{*}\right) \in\left[\eta_{k}-b_{k}^{*} \frac{\Delta_{K}}{K_{k}^{* 2}}, \eta_{k}+b_{k}^{*} \frac{\Delta_{K}}{K_{k}^{* 2}}\right],
$$

for all $k \in \mathbf{N}_{0}$, which is trivially bounded, provided that the disturbance sequence is bounded.

(iii) A positive carrying capacity is obtained from the controller synthesis if $\left(K_{k}^{*}\right)^{-1}>\left(\omega_{k} x_{k}-\right.$ $\left.f_{k}\right) / t_{k} x_{k}=\left(1 / t_{k}\right)\left(\bar{\eta}_{k} /\left(1-a_{k}\right)+\left(a_{k}-a_{k}^{*}\right) / x_{k}\right)$, when $\eta_{k}$ is unknown but $\bar{\eta}_{k} \geq\left|\eta_{k}\right|$ is known. The control parameter $t_{k} \in(0,1]$ and the calculated $K_{k}$ from the control law are positive if $t_{k}$ is calculated satisfying $\Delta_{K} \in\left[0, K_{k}^{*}-\bar{\eta}_{k} /\left(1-a_{k}\right)+\left(a_{k}^{*}-a_{k}\right) / x_{k}\right)$ with $\bar{\eta}_{k} \in$ $\left[0,\left(1-a_{k}\right)\left(K_{k}^{*}+\left(a_{k}-a_{k}^{*}\right) / x_{k}\right)\right)$. 
Proof. (i) Note that

$$
\begin{aligned}
t_{k} \in\left[1-\frac{\Delta_{K}}{K_{k}^{*}}, 1+\frac{\Delta_{K}}{K_{k}^{*}}\right] & \Longleftrightarrow\left(1-a_{k}\right) \in\left[\frac{K_{k}^{*}\left(1-a_{k}^{*}\right)}{K_{k}^{*}+\Delta_{K}}, \frac{K_{k}^{*}\left(1-a_{k}^{*}\right)}{K_{k}^{*}-\Delta_{K}}\right] \\
& \Longleftrightarrow 1-\frac{K_{k}^{*}\left(1-a_{k}^{*}\right)}{K_{k}^{*}+\Delta_{K}} \geq a_{k} \geq 1-\frac{K_{k}^{*}\left(1-a_{k}^{*}\right)}{K_{k}^{*}-\Delta_{K}},
\end{aligned}
$$

which yields directly Property (i) since $b_{k}=1-a_{k}$.

(ii) One gets from (2.6)-(2.8) and Property (i) that

$$
\begin{aligned}
\varepsilon_{k+1} & =s_{k+1}-s_{k+1}^{*}=a_{k} \varepsilon_{k}+\left(a_{k}-a_{k}^{*}\right) s_{k}^{*}+\left(t_{k}-1\right) b_{k}^{*} r_{k}+\eta_{k} \\
& \in\left[a_{k} \varepsilon_{k}+\left(a_{k}-a_{k}^{*}\right) s_{k}^{*}-\frac{\Delta_{K}}{K_{k}^{*}} b_{k}^{*} r_{k}+\eta_{k}, a_{k} \varepsilon_{k}+\left(a_{k}-a_{k}^{*}\right) s_{k}^{*}+\frac{\Delta_{K}}{K_{k}^{*}} b_{k}^{*} r_{k}+\eta_{k}\right],
\end{aligned}
$$

and the result follows.

(iii) The first part follows with the replacement of $\eta_{k} \rightarrow \bar{\eta}_{k} \geq\left|\eta_{k}\right|$ in (2.9). The second part follows directly by replacing the admissibility domain of the controller parameter $t_{k}$ in the above constraint.

\section{Adaptive control}

For the case when the parameters of the BHE are unknown, an estimation scheme with adaptation dead zone for robust closed-loop stabilization is incorporated (see, e.g., $[12,13,15,16]$ ). Such a mechanism governs the evolution of the IBHE and it is now discussed from a theoretical point of view. It will then corroborate, through numerical simulation, the potential usefulness of numerical and adaptive techniques as previously tested in some ecological models (see, e.g., [17-19]). One takes the advantage that, since the parameters fulfill the constraint $a_{k}+b_{k}=1$, for all $k \in \mathbf{N}_{0}$, the same constraint might be introduced in the estimation scheme, what reduces it to the estimation of one parameter only. A modification would consist of estimating both sequences of parameters separately. In that case, the estimation is performed without taking into account the parametrical constraint at the expense of an increase in computation time and memory storage. However, it may be proven that all the properties of boundedness and convergence of the estimates still hold if existing parametrical constraints are not taken into account, provided that each parameter is updated with its corresponding component in the set of measured data (see, e.g., $[12,13,15])$. In a first step, a simple procedure is developed for the nominal BHE through controlling its inverse, and then an extension is made to prevent a good operation in the presence of additive disturbances. A relative dead zone to the estimation scheme is added to prevent potential instability caused by those disturbances.

\subsection{Adaptive control approach based on the intrinsic growth rate estimate}

\section{Additive disturbances}

The deviations of the intrinsic growth rate with respect to a certain unknown constant value $\mu=a^{-1}$, implying $b=1-a$, are incorporated within a disturbance sequence $\left\{\eta_{k}\right\}_{0}^{\infty}$ which can 
also include other unstructured disturbance contributions in a sequence $\left\{\eta_{k}^{0}\right\}_{0}^{\infty}$ :

$$
\eta_{k}:=\left(a_{k}-a\right) s_{k}+\left(b_{k}-b\right) u_{k}+\eta_{k}^{0}=\left(a_{k}-a\right)\left(s_{k}-u_{k}\right)+\eta_{k}^{0}
$$

and $u_{k}=K_{k}^{-1}$. In this way, the nominal parameter vector of the IBHE, namely, $\theta=(a, b)^{T}$, is constant, which then facilitates an updating algorithm that has an identical structure for the parameter estimates as for the parametrical errors. The IBHE, its estimate, the identification error, the reference IBHE, the tracking error, and the parameter-adaptive law are, respectively, as follows.

IBHE

$$
s_{k+1}=a s_{k}+b u_{k}+\eta_{k}=a\left(s_{k}-u_{k}\right)+u_{k}+\eta_{k}
$$

If $\eta_{k} \equiv 0$, the resulting particular case of (3.2) is called the nominal IBHE, and its corresponding inverse equation, whose solution is the sequence $\left\{x_{k} \equiv s_{k}^{-1}\right\}_{0}^{\infty}$, is called the nominal BHE.

Estimate of the IBHE

$$
\widehat{s}_{k+1}=\widehat{a}_{k} s_{k}+\widehat{b}_{k} u_{k}=\widehat{a}_{k}\left(s_{k}-u_{k}\right)+u_{k}
$$

where $\widehat{a}_{k}$ and $\widehat{b}_{k}=1-\widehat{a}_{k}$ are the estimates of $a$ and $b$ at the $k$ th sample.

\section{Identification error}

$$
e_{k+1}:=s_{k+1}-\widehat{s}_{k+1}=-\tilde{a}_{k} s_{k}-\tilde{b}_{k} u_{k}+\eta_{k}=-\tilde{a}_{k}\left(s_{k}-u_{k}\right)+\eta_{k}
$$

where $\tilde{a}_{k}:=\widehat{a}_{k}-a, \widehat{b}_{k}:=\widehat{b}_{k}-b=a-\widehat{a}_{k}=-\widetilde{a}_{k}$ are the parametrical errors. Note that although $\eta_{k}$ is unknown, the identification error is available for measurement through the first identity in (3.4).

\section{Reference IBHE}

It is assumed to be time invariant as the nominal IBHE, namely,

$$
s_{k+1}^{*}=a^{*} s_{k}^{*}+b^{*} r_{k}=a^{*}\left(s_{k}^{*}-r_{k}\right)+r_{k}
$$

with $b^{*}=1-a^{*}$ and $r_{k}=\left(K_{k}^{*}\right)^{-1}$, is the reference environment carrying capacity inverse.

Tracking error

$$
\varepsilon_{k+1}:=s_{k+1}^{*}-s_{k+1}=\left(s_{k+1}^{*}-\widehat{s}_{k+1}\right)+\left(\widehat{s}_{k+1}-s_{k+1}\right)=s_{k+1}^{*}-\widehat{s}_{k+1}-e_{k+1} .
$$


Adaptation Algorithm 1:

$$
\widehat{a}_{k+1}=\widehat{a}_{k}+\frac{\sigma_{k}\left(s_{k}-u_{k}\right) e_{k+1}}{1+\gamma_{k}\left(s_{k}-u_{k}\right)^{2}}, \quad \widehat{a}_{0} \in(0,1), \forall k \in \mathbf{N}_{0},
$$

is a recursive estimation of the intrinsic growth rate, where $\left\{\sigma_{k}\right\}_{0}^{\infty}$ and $\left\{\gamma_{k}\right\}_{0}^{\infty}$ are real sequences satisfying $\sigma_{k} \geq 0, \gamma_{k}>0$ to be specified later for purposes of convergence of the algorithm. The control law has the same structure as (2.7)-(2.9) by replacing the true parameters by its estimates, and by deleting the correcting sequence $\omega_{k}$. Instability drawbacks due to disturbances will be dealt with by using a specific dead-zone updating rule for the sequence $\sigma_{k} \geq 0$ in the parameter-adaptive algorithm.

Adaptive control law:

$$
u_{k}=t_{k} r_{k}+f_{k} s_{k}=\frac{1}{1-\widehat{a}_{k}}\left[\left(1-a_{k}^{*}\right) r_{k}+\left(a_{k}^{*}-\widehat{a}_{k}\right) s_{k}\right]
$$

with $r_{k}=\left(K_{k}^{*}\right)^{-1}$ with the sample-dependent controller parameters

$$
f_{k}=\frac{a_{k}^{*}-\widehat{a}_{k}}{1-\widehat{a}_{k}}, \quad t_{k}=\frac{1-a_{k}^{*}}{1-\widehat{a}_{k}}
$$

The following result concerning useful technical relationships in between the IBHE solution, its estimated IBHE, and the reference IBHE independent of the parameter adaptive algorithm is immediate.

Proposition 3.1. The adaptive control law (3.8)-(3.9) leads to $\widehat{s}_{k}=s_{k}^{*}$ (i.e., the estimated solution equalizes the reference solution), $\varepsilon_{k}=-e_{k}$ (i.e., the tracking error equalizes the minus-identification error), for all $k \in \mathbf{N}$, for any finite $s_{0}, \widehat{s}_{0}=s_{0}^{*}$. The above results are independent of the parameteradaptive algorithm.

Proof. Proceed, by induction, by assuming $\widehat{s}_{k}=s_{k}^{*}$ for any given $k \in \mathbf{N}_{0}$. Then one gets, from (3.8)-(3.9) into (3.3) by using $\widehat{b}_{k}=1-\widehat{a}_{k}$,

$$
\begin{aligned}
\widehat{s}_{k+1} & =\widehat{a}_{k} s_{k}+\left(1-\widehat{a}_{k}\right)\left(t_{k} r_{k}+f_{k} s_{k}\right)=\left[\widehat{a}_{k}\left(1-f_{k}\right)+f_{k}\right] s_{k}+\left(1-\widehat{a}_{k}\right) t_{k} r_{k} \\
& =a_{k}^{*} s_{k}+b_{k}^{*} r_{k}=s_{k+1}^{*}
\end{aligned}
$$

if $s_{k}=s_{k}^{*}$, and then using (3.6),

$$
\varepsilon_{k+1}=\left(s_{k+1}^{*}-\widehat{s}_{k+1}\right)+\left(\widehat{s}_{k+1}-s_{k+1}\right)=\left(\widehat{s}_{k+1}-s_{k+1}\right)=-e_{k+1}, \quad \forall k \in \mathbf{N}_{0},
$$

what completes the proof.

\subsection{Boundedness and convergence results of the adaptation algorithm}

In the following, the boundedness and convergence properties of the adaptation algorithm are investigated in the subsequent result. 
Theorem 3.2. Assume that a sequence $\left\{\bar{\eta}_{k}\right\}_{0}^{\infty}$ satisfying $\bar{\eta}_{k} \geq\left|\eta_{k}\right|$, for all $k \in \mathbf{N}_{0}$, is known that the adaptation algorithm (3.7) is updated by choosing $\gamma_{k} \geq \varsigma_{0}$, and

$$
\sigma_{k}= \begin{cases}0 & \text { if }\left|e_{k+1}\right| \leq s \bar{\eta}_{k^{\prime}} \\ \frac{2 \gamma_{k}\left(s-1-s_{1}\right)}{s} & \text { if }\left|e_{k+1}\right|>s \bar{\eta}_{k^{\prime}}\end{cases}
$$

for all $k \in \mathbf{N}_{0}$, for some prefixed real constants $\varsigma>1, \varsigma-1>\varsigma_{0}>0$, and $\varsigma_{1} \in\left[\varsigma_{0}, \varsigma-1\right)$. Then the following properties hold irrespective of the control law.

(i) The sequences $\left\{\widehat{a}_{k}\right\}_{0}^{\infty},\left\{\left(\sigma_{k} /\left(1+\gamma_{k}\left(s_{k}-u_{k}\right)^{2}\right)\right)^{1 / 2}\left|e_{k+1}\right|\right\}_{0}^{\infty}$, and $\left\{\left(\sigma_{k} /\left(1+\gamma_{k}\left(s_{k}-u_{k}\right)^{2}\right)\right) e_{k+1}^{2}\right\}_{0}^{\infty}$ are bounded and have limits $\widehat{a}_{\infty}<\infty$, and zero, respectively.

(ii) There exist the subsequent limits

$$
\begin{aligned}
\lim _{k \rightarrow \infty}\left(\sum_{i=0}^{k} \frac{\sigma_{i} \eta_{i}^{2}}{1+\gamma_{i}\left(s_{i}-u_{i}\right)^{2}}\right) & \leq \lim _{k \rightarrow \infty}\left(\sum_{i=0}^{k} \frac{\sigma_{i} \bar{\eta}_{i}^{2}}{1+\gamma_{i}\left(s_{i}-u_{i}\right)^{2}}\right) \\
& \leq \lim _{k \rightarrow \infty}\left(\sum_{i=0}^{k} \frac{\sigma_{i} e_{i+1}^{2}}{1+\gamma_{i}\left(s_{i}-u_{i}\right)^{2}}\right)<\infty, \\
\lim _{k \rightarrow \infty}\left(\sum_{i=0}^{k}\left(\frac{\sigma_{i} \eta_{i}^{2}}{1+\gamma_{i}\left(s_{i}-u_{i}\right)^{2}}\right)^{1 / 2}\right) & \leq \lim _{k \rightarrow \infty}\left(\sum_{i=0}^{k}\left(\frac{\sigma_{i} \bar{\eta}_{i}^{2}}{1+\gamma_{i}\left(s_{i}-u_{i}\right)^{2}}\right)^{1 / 2}\right) \\
& \leq \lim _{k \rightarrow \infty}\left(\left(\frac{\sigma_{i} e_{i+1}^{2}}{1+\gamma_{i}\left(s_{i}-u_{i}\right)^{2}}\right)^{1 / 2}\right)<\infty .
\end{aligned}
$$

Proof. Let $\left\{V_{k}\right\}_{0}^{\infty}$ be defined by $V_{k}:=\tilde{a}_{k}^{2}$. Note, by subtracting $a$ in both sides of (3.7), that $\tilde{a}_{k+1}=\tilde{a}_{k}+\sigma_{k} \varphi_{k} e_{k+1} /\left(1+\gamma_{k} \varphi_{k}^{2}\right)$, where $\varphi_{k}:=s_{k}-u_{k}$. Since

$$
\tilde{a}_{k} \varphi_{k}=\eta_{k}-e_{k+1} \leq s \bar{\eta}_{k}-e_{k+1}
$$

from (3.4), for any absolute upper-bound $\bar{\eta}_{k} \geq\left|\eta_{k}\right|$, one gets

$$
\begin{aligned}
\Delta V_{k}:=\tilde{a}_{k+1}^{2}-\tilde{a}_{k}^{2} & \leq \frac{\sigma_{k}^{2} \varphi_{k}^{2} e_{k+1}^{2}}{\left(1+\gamma_{k} \varphi_{k}^{2}\right)^{2}}+\frac{2 \sigma_{k}\left|e_{k+1}\right|\left(\bar{\eta}_{k}-\left|e_{k+1}\right|\right)}{1+\gamma_{k} \varphi_{k}^{2}} \\
& =\frac{\sigma_{k}\left|e_{k+1}\right|}{1+\gamma_{k} \varphi_{k}^{2}}\left(\frac{\sigma_{k} \varphi_{k}^{2}\left|e_{k+1}\right|}{1+\gamma_{k} \varphi_{k}^{2}}-2\left(\left|e_{k+1}\right|-\bar{\eta}_{k}\right)\right) .
\end{aligned}
$$

From (3.15), $\sigma_{k}=0 \Rightarrow \Delta V_{k}=0$. Assume $\left|e_{k+1}\right|>\varsigma \bar{\eta}_{k}$ so that for any real $\varsigma>1$, one gets, from (3.15),

$$
\Delta V_{k} \leq \frac{\sigma_{k} e_{k+1}^{2}}{1+\gamma_{k} \varphi_{k}^{2}}\left(\frac{\sigma_{k} \varphi_{k}^{2}}{1+\gamma_{k} \varphi_{k}^{2}}-2\left(1-\varsigma^{-1}\right)\right) \leq-\frac{2 \sigma_{k} \zeta_{1} e_{k+1}^{2}}{\varsigma\left(1+\gamma_{k} \varphi_{k}^{2}\right)} \leq 0
$$


for all $k \in \mathbf{N}_{0}$, provided that $\gamma_{k}>0$ and $\sigma_{k}$ satisfies $0 \leq \sigma_{k} \varphi_{k}^{2} /\left(1+\gamma_{k} \varphi_{k}^{2}\right) \leq \sigma_{k} / \gamma_{k} \leq 2\left(\varsigma-1-\varsigma_{1}\right) / \varsigma_{0}$ for any real $\varsigma_{1} \in\left[\varsigma_{0}, \varsigma^{-1}\right)$. Then $V_{k} \leq V_{0}<\infty$, what implies that the sequences $\left\{\left|\Delta V_{k}\right|\right\}_{0}^{\infty},\left\{\tilde{a}_{k}\right\}_{0}^{\infty}$ and $\left\{\widehat{a}_{k}\right\}_{0}^{\infty}$, and

$$
\left\{\left(\frac{\sigma_{k}}{1+\gamma_{k}\left(s_{k}-u_{k}\right)^{2}}\right)^{1 / 2}\left|e_{k+1}\right|\right\}_{0}^{\infty}, \quad\left\{\left(\frac{\sigma_{k}}{1+\gamma_{k}\left(s_{k}-u_{k}\right)^{2}}\right)^{1 / 2}\left|\bar{\eta}_{k}\right|\right\}_{0}^{\infty}
$$

are uniformly bounded and there exist $\operatorname{limits}_{\lim _{k \rightarrow \infty}}\left(\left|\Delta V_{k}\right|\right)=\lim _{k \rightarrow \infty}\left(\sigma_{k} e_{k+1}^{2} /\left(1+\gamma_{k}\left(s_{k}-\right.\right.\right.$ $\left.\left.\left.u_{k}\right)^{2}\right)\right)=0, \lim _{k \rightarrow \infty}\left(V_{k}\right)=V_{\infty} \leq V_{0}<\infty, \lim _{k \rightarrow \infty}\left(\widehat{a}_{k}\right)=\widehat{a}_{\infty}<\infty$, and $\lim _{k \rightarrow \infty}\left(\widetilde{a}_{k}\right)=\tilde{a}_{\infty}<\infty$. $\lim _{k \rightarrow \infty}\left(\sigma_{k} e_{k+1}^{2} /\left(1+\gamma_{k}\left(s_{k}-u_{k}\right)^{2}\right)\right)=0 \Rightarrow \lim _{k \rightarrow \infty}\left(\left(\sigma_{k} /\left(1+\gamma_{k}\left(s_{k}-u_{k}\right)^{2}\right)\right)^{1 / 2}\left|e_{k+1}\right|\right)=0$ so that $\lim _{k \rightarrow \infty}\left(\left(\sigma_{k} /\left(1+\gamma_{k}\left(s_{k}-u_{k}\right)^{2}\right)\right)^{1 / 2}\left|\bar{\eta}_{k}\right|\right)=0$ since either $\sigma_{k} \geq 2 \varsigma_{0}\left(s-1-\varsigma_{1}\right) / \varsigma$ or $\sigma_{k}=0$, for all $k \in \mathbf{N}_{0}$, and Property (i) is proven. Since $V_{k}$ converges to a finite limit, Property (ii) follows from

$$
\sum_{i=0}^{k} \frac{\sigma_{k} \bar{\eta}_{i}^{2}}{1+\gamma_{k}\left(s_{k}-u_{k}\right)^{2}} \leq \sum_{i=0}^{k} \frac{\sigma_{k} e_{k+1}^{2}}{1+\gamma_{k}\left(s_{k}-u_{k}\right)^{2}}=\sum_{i=0}^{k}\left|\Delta V_{i}\right|=V_{0}-V_{k+1} \leq V_{0}-V_{\infty}<\infty
$$

as well as the boundedness of the square of each element of the sequence associate with the above series.

The boundedness of the estimates and estimation and tracking errors as well as the convergence of the estimates to finite limits are crucial issues to formulate a well-posed problem. The relative dead zone of the algorithm (3.12) is relevant for adaptive stabilization under external disturbances. The interpretation is direct; if the identification error is small related to the known upper bound of the disturbances, the estimation is frozen (i.e., stopped). The reason is that the contribution to the identification error of the parameterized part, which is being updated, may be smaller than that of the disturbance and then to maintain the estimation, updating may lead to instability. The incorporation of relative adaptation dead zones into the estimation scheme is a standard mechanism to cope against adaptive instability since the sequence $\left\{V_{k}\right\}_{0}^{\infty}$ is bounded, see, for instance, $[12,13,15]$. Otherwise, the estimates computations and then the control law are unfeasible. If Assumption 2.1 is satisfied, then a projection method may be used for the estimates to guarantee that they lie inside its definition domain. Theorem 3.2 is then extended as follows.

Theorem 3.3. If both the nominal and current intrinsic growth rates satisfy Assumption 2.1, then there exist real constants $\varepsilon_{\mu} \in \mathbf{R}_{0}^{+}, \varepsilon_{1}>1$, such that

$$
a, a_{k} \in\left[\frac{1}{1+\varepsilon_{1} \varepsilon_{\mu}}, \frac{1}{1+\varepsilon_{\mu}}\right], \quad \forall k \in \mathbf{N}_{0},
$$

and the above adaptation algorithm is replaced with the set of modified recursive equations (3.20)-(3.22) below as to include projection of the estimates on an admissibility domain.

Adaptation Algorithm 2

It consists of two steps, namely, a priori and a posteriori estimations as follows. 
A priori estimation:

$$
\widehat{a}_{k+1}^{0}=\widehat{a}_{k+1}+\frac{\sigma_{k}\left(s_{k}-u_{k}\right) e_{k+1}}{1+\gamma_{k}\left(s_{k}-u_{k}\right)^{2}}, \quad \widehat{a}_{0} \in(0,1), \forall k \in \mathbf{N}_{0},
$$

being updated by choosing $\gamma_{k} \geq \varsigma_{0}$, and

$$
\sigma_{k}= \begin{cases}0 & \text { if }\left|e_{k+1}\right| \leq s \bar{\eta}_{k^{\prime}}^{0} \\ \frac{2 \gamma_{k}\left(s-1-s_{1}\right)}{s} & \text { if }\left|e_{k+1}\right|>s \bar{\eta}_{k}^{0}: s\left(\bar{\eta}_{k}+\left(1-\frac{\widehat{a}_{k}}{\widehat{a}_{k}^{0}}\right) \widehat{a}_{k}^{0}\left(s_{k}-u_{k}\right)\right),\end{cases}
$$

for all $k \in \mathbf{N}_{0}$, for some prefixed real constants $\varsigma>1, \varsigma_{0}>0$, and $\varsigma_{1} \in\left[\varsigma_{0}, \varsigma-1\right)$.

A posteriori estimation via projection on the interval $\left[1 /\left(1+\varepsilon_{1} \varepsilon_{\mu}\right), 1 /\left(1+\varepsilon_{\mu}\right)\right]$ :

$$
\widehat{a}_{k+1}= \begin{cases}\frac{1}{1+\varepsilon_{1} \varepsilon_{\mu}} & \text { if } \widehat{a}_{k+1}^{0} \leq \frac{1}{1+\varepsilon_{1} \varepsilon_{\mu}}, \\ \widehat{a}_{k+1}^{0} & \text { if } \widehat{a}_{k+1}^{0} \in\left(\frac{1}{1+\varepsilon_{1} \varepsilon_{\mu}}, \frac{1}{1+\varepsilon_{\mu}}\right), \\ \frac{1}{1+\varepsilon_{\mu}} & \text { if } \widehat{a}_{k+1}^{0} \geq \frac{1}{1+\varepsilon_{\mu}} .\end{cases}
$$

Then Theorem 3.2(i)-(ii) holds. In addition, similar properties hold by replacing

$$
\begin{aligned}
& \eta_{k} \longrightarrow \eta_{k}^{0}=\eta_{k}+\left(1-\frac{\widehat{a}_{k}}{\widehat{a}_{k}^{0}}\right) \widehat{a}_{k}^{0}\left(s_{k}-u_{k}\right), \quad \bar{\eta}_{k} \longrightarrow \bar{\eta}_{k}^{0}=\bar{\eta}_{k}+\left(1-\frac{\widehat{a}_{k}}{\widehat{a}_{k}^{0}}\right) \widehat{a}_{k}^{0}\left(s_{k}-u_{k}\right), \\
& e_{k+1}[\text { A posteriori identification error }]:=s_{k+1}-\widehat{s}_{k+1}=s_{k+1}-\widehat{a}_{k}\left(s_{k}-u_{k}\right) \\
& \longrightarrow e_{k+1}^{0}[\text { A priori identification error }]:=s_{k+1}-\widehat{s}_{k+1}^{0}=s_{k+1}-\widehat{a}_{k}^{0}\left(s_{k}-u_{k}\right) .
\end{aligned}
$$

Proof. Define the sequence

$$
0 \leq \Delta V_{k}^{0}:=\left(\tilde{a}_{k+1}^{0}\right)^{2}-\tilde{a}_{k}^{2} \leq \frac{\sigma_{k}\left|e_{k+1}\right|}{1+\gamma_{k} \varphi_{k}^{2}}\left(\frac{\sigma_{k} \varphi_{k}^{2}\left|e_{k+1}\right|}{1+\gamma_{k} \varphi_{k}^{2}}-2\left(\left|e_{k+1}\right|-\bar{\eta}_{k}^{0}\right)\right) \leq 0
$$

from (3.20)-(3.21) using a close development to that in Theorem 3.2 so that it is monotonically decreasing

$$
\Delta V_{k}:=\tilde{a}_{k+1}^{2}-\tilde{a}_{k}^{2}=\tilde{a}_{k+1}^{2}-\left(\tilde{a}_{k+1}^{0}\right)^{2}-\left|\Delta V_{k}^{0}\right| \leq-\left(1-\left(\frac{\widehat{a}_{k+1}}{\widehat{a}_{k+1}^{0}}\right)^{2}\right)\left(\tilde{a}_{k+1}^{0}\right)^{2}-\left|\Delta V_{k}^{0}\right| \leq 0
$$

for all $k \in \mathbf{N}_{0}$ since $0<\widehat{a}_{k+1} / \widehat{a}_{k+1}^{0} \leq 1$, for all $k \in \mathbf{N}_{0}$, from the projection part (3.22) of the estimation algorithm. Then $\left\{V_{k}\right\}_{0}^{\infty}$ is bounded and converges to a finite limit, and $\left\{\Delta V_{k}\right\}_{0}^{\infty}$ is also bounded and converges to zero. Also $\left|\Delta V_{k}^{0}\right|=\left(1-\widehat{a}_{k+1} / \widehat{a}_{k+1}^{0}\right)<1$ for all $k \in \mathbf{N}_{0}$, and 
$\left|\Delta V_{k}^{0}\right|=\left(1-\widehat{a}_{k+1} / \widehat{a}_{k+1}^{0}\right)<1$. Since $\Delta V_{k}^{0}:=\left(\tilde{a}_{k+1}^{0}\right)^{2}-\tilde{a}_{k}^{2}=0$, for all $k \in \mathbf{N}_{0}$, then there exists a finite limit $\lim _{k \rightarrow \infty}\left(\sum_{i=0}^{k}\left|\Delta V_{i}^{0}\right|\right)<\infty$, then $\left(\left(\tilde{a}_{k+1}^{0}\right)^{2}-\tilde{a}_{k}^{2}\right)$ converges to a finite limit and $\left|\tilde{a}_{k}^{0}\right|$ converges since $\left|\tilde{a}_{k}\right|$ converges (since $V_{k}$ converges) to finite limits as $k \rightarrow \infty$. Thus Theorem 3.2(i)-(ii) follows. On the other hand, note that

$$
\begin{aligned}
& e_{k+1} \text { [A posteriori identification error] }:=s_{k+1}-\widehat{s}_{k+1}=\eta_{k}-\tilde{a}_{k}\left(s_{k}-u_{k}\right) \\
& =e_{k+1}^{0}+\left(\widehat{a}_{k}^{0}-\widehat{a}_{k}\right)\left(s_{k}-u_{k}\right)=\eta_{k}^{0}-\tilde{a}_{k}^{0}\left(s_{k}-u_{k}\right), \\
& e_{k+1}^{0}\left[\text { A priori identification error] }:=s_{k+1}-\widehat{s}_{k+1}^{0}=\eta_{k}-\tilde{a}_{k}^{0}\left(s_{k}-u_{k}\right)\right. \\
& =e_{k+1}+\left(\widehat{a}_{k}-\widehat{a}_{k}^{0}\right)\left(s_{k}-u_{k}\right)=\eta_{k}^{0}+\left(1-\frac{\widehat{a}_{k}}{\widehat{a}_{k}^{0}}\right)\left(2 \widehat{a}_{k}^{0}-a\right)\left(s_{k}-u_{k}\right) .
\end{aligned}
$$

Since the normalized sequences $\left\{\left(\sigma_{k} /\left(1+\gamma_{k} \varphi_{k}^{2}\right)\right)^{1 / 2}\left|e_{k+1}\right|\right\}_{0}^{\infty}$ and $\left\{\left(\sigma_{k} /\left(1+\gamma_{k} \varphi_{k}^{2}\right)\right)^{1 / 2} \bar{\eta}_{k}^{0}\right\}_{0}^{\infty}$ are bounded, it follows that the reported replacements may be performed leading to Theorem 3.3(iii).

\subsection{Some practical design constraints}

The tracking error of the solutions of the IBHE and the reference IBHE are given by the set of equivalent expressions:

$$
\varepsilon_{k+1}=s_{k+1}^{*}-s_{k+1}=s_{k+1}^{*}-\widehat{s}_{k+1}-e_{k+1}=s_{k+1}^{*}-\widehat{s}_{k+1}+\tilde{a}_{k}\left(s_{k}-u_{k}\right)-\eta_{k} .
$$

The solution of the IBHE is equivalently calculated either as a function of the nominal parameters $a$ and $b=1-a$ or as a function of their estimates as follows, by considering the adaptive control law (3.8):

$$
\begin{aligned}
s_{k+1} & =a\left(s_{k}-u_{k}\right)+u_{k}+\eta_{k}=\widehat{s}_{k+1}+e_{k+1}=\widehat{a}_{k}\left(s_{k}-u_{k}\right)+u_{k}+\eta_{k}-\tilde{a}_{k}\left(s_{k}-u_{k}\right) \\
& =\widehat{s}_{k+1}+e_{k+1}=\widehat{a}_{k}\left(s_{k}-u_{k}\right)+u_{k}+\eta_{k}-\tilde{a}_{k}\left(s_{k}-u_{k}\right) \\
& =\widehat{a}_{k} s_{k}+\left[\left(1-a_{k}^{*}\right) r_{k}+\left(a_{k}^{*}-\widehat{a}_{k}\right) s_{k}\right]+e_{k+1} \\
& =a_{k}^{*} s_{k}+\left(1-a_{k}^{*}\right) r_{k}+e_{k+1} .
\end{aligned}
$$

It is important to discuss when the adaptive control law has no division by zero with the property being numerically robust. This translates, in practice, to $\widehat{a}_{k} \neq 1$ being sufficiently deviated from unity and $\left\{s_{k}\right\}_{0}^{\infty}$ with all its elements positive and sufficiently deviated from zero. This guarantees that the BHE does not diverge when driven by an appropriate nonzero finitely upper-bounded environment carrying capacity. More formally, a reasonable assumption for practical design purposes is to extend the assumption of Theorem 3.3 to also consider the same admissibility range referred to for the reference intrinsic growth-rate inverse and the estimated intrinsic growth rate. 
Assumption 3.4. $a, a_{k}, \widehat{a}_{k}, a_{k}^{*} \in\left[1 /\left(1+\varepsilon_{1} \varepsilon_{\mu}\right), 1 /\left(1+\varepsilon_{\mu}\right)\right]$, for all $k \in \mathbf{N}_{0}$, and some prefixed real constants $\varepsilon_{\mu}>0$ and $\varepsilon_{1}>1$. A direct calculation with Assumption 3.4 yields

$$
\begin{aligned}
\left(1-\widehat{a}_{k}\right) & \in\left[\frac{\varepsilon_{\mu}}{1+\varepsilon_{\mu}}, \frac{\varepsilon_{1} \varepsilon_{\mu}}{1+\varepsilon_{1} \varepsilon_{\mu}}\right] \subset(0,1), \quad\left(\frac{1}{1-\widehat{a}_{k}}\right) \in\left[1+\frac{1}{\varepsilon_{1} \varepsilon_{\mu}}, 1+\frac{1}{\varepsilon_{\mu}}\right], \\
\left|a_{k}^{*}-\widehat{a}_{k}\right| & \leq \frac{\varepsilon_{\mu}\left(\varepsilon_{1}-1\right)}{\left(1+\varepsilon_{1} \varepsilon_{\mu}\right)\left(1+\varepsilon_{\mu}\right)}, \\
\left(a_{k}^{*}-\widehat{a}_{k}\right) & \leq\left[-\frac{\left(\varepsilon_{1}-1\right) \varepsilon_{\mu}}{\left(1+\varepsilon_{1} \varepsilon_{\mu}\right)\left(1+\varepsilon_{\mu}\right)}, \frac{\left(\varepsilon_{1}-1\right) \varepsilon_{\mu}}{\left(1+\varepsilon_{1} \varepsilon_{\mu}\right)\left(1+\varepsilon_{\mu}\right)}\right],
\end{aligned}
$$

while similar expressions follow for replacements involving any two elements of the quadruplet $\left(a, a_{k}, \hat{a}_{k}, a_{k}^{*}\right)$ as follows:

$$
\begin{aligned}
& \left(\frac{1+\varepsilon_{\mu}}{1+\varepsilon_{1} \varepsilon_{\mu}}\right)\left[\varepsilon_{1} r_{k}+\frac{\varepsilon_{1}-1}{1+\varepsilon_{\mu}} s_{k}\right] \geq u_{k}=\frac{1}{1-\widehat{a}_{k}}\left[\left(1-a_{k}^{*}\right) r_{k}+\left(\widehat{a}_{k}-a_{k}^{*}\right) s_{k}\right] \\
& \quad \geq\left(\frac{1+\varepsilon_{\mu}}{1+\varepsilon_{1} \varepsilon_{\mu}}\right)\left[\varepsilon_{1}\left(r_{k}-\frac{1}{1+\varepsilon_{\mu}} s_{k}\right)+\frac{1}{1+\varepsilon_{\mu}} s_{k}\right] \geq\left(\frac{1+\varepsilon_{\mu}}{1+\varepsilon_{1} \varepsilon_{\mu}}\right)\left[\varepsilon_{1}\left(r_{k}-s_{k}\right)+\frac{1}{1+\varepsilon_{\mu}} s_{k}\right] .
\end{aligned}
$$

The above expressions (3.29)-(3.30) lead to the following result referring to explicit positive lower and upper bounds of $u_{k}=K_{k}^{-1}=\left(K_{k}^{*}+\widetilde{K}_{k}\right)^{-1}$ so that $u_{k}^{-1}=K_{k}^{*}+\widetilde{K}_{k}=K_{k}^{*}+r_{k}^{-1}$ since $r_{k}=\left(K_{k}^{*}\right)^{-1}$.

Proposition 3.5. Assume $s_{k}<\varepsilon_{1}\left(1+\varepsilon_{\mu}\right) /\left(\varepsilon_{1}-1\right) K_{k^{\prime}}^{*}$ for all $k \in \mathbf{N}_{0}$. Then the following constraints hold:

$$
\begin{gathered}
\frac{1+\varepsilon_{\mu}}{\varepsilon_{1}\left(1+\varepsilon_{\mu}\right)\left(K_{k}^{*}\right)^{-1}+\left(\varepsilon_{1}-1\right) s_{k}} \leq \frac{1}{u_{k}} \leq \frac{1+\varepsilon_{\mu}}{\varepsilon_{1}\left(1+\varepsilon_{\mu}\right)\left(\left(K_{k}^{*}\right)^{-1}-s_{k}\right)+s_{k}}, \\
\frac{1+\varepsilon_{\mu}}{\varepsilon_{1}\left(1+\varepsilon_{\mu}\right)\left(K_{k}^{*}\right)^{-1}+\left(\varepsilon_{1}-1\right) s_{k}}-K_{k}^{*} \leq \widetilde{K}_{k} \leq \frac{1+\varepsilon_{\mu}}{\varepsilon_{1}\left(1+\varepsilon_{\mu}\right)\left(K_{k}^{*}\right)^{-1}-\left(\varepsilon_{1}-1\right) s_{k}}-K_{k}^{*} .
\end{gathered}
$$

Proof. It follows directly from (3.29)-(3.30). The necessary condition $s_{k}<\varepsilon_{1}\left(1+\varepsilon_{\mu}\right) /\left(\varepsilon_{1}-1\right) K_{k}^{*}$ is needed to keep $\left(\varepsilon_{1}\left(1+\varepsilon_{\mu}\right)\left(K_{k}^{*}\right)^{-1}-\left(\varepsilon_{1}-1\right) s_{k}\right)>0$ in the right-hand-side upper bound of $\widetilde{K}_{k}$, for all $k \in \mathbf{N}_{0}$ in (3.32).

\subsection{Closed-loop stability}

The boundedness and convergence properties of the parameter estimates have been proven. In the following, the closed-loop stability is proven under a condition of slow growing of the disturbances with respect to the solution of the IBHE if such a disturbance is unknown. In particular, it is assumed that $\bar{\eta}_{k}=O\left[\operatorname{Max}_{0 \leq i \leq k}\left(s_{i}\right)\right]$ with known constants of the related linear upper bound and with sufficiently slow growth of $\bar{\eta}_{k}$ with respect to $\operatorname{Max}_{0 \leq i \leq k}\left(s_{i}\right)$. This is a reasonable assumption used in the background literature since a complete lack of knowledge of disturbances makes impossible the stabilization in the general case (see, e.g., $[12,13,15]$ and references therein). 
Assumption 3.6. There exist known finite nonnegative real constants $\lambda_{1,2}$ such that

$$
\bar{\eta}_{k} \leq \lambda_{1}+\lambda_{2} \operatorname{Max}_{0 \leq i \leq k}\left(s_{i}\right) .
$$

Then, from (3.28b), (3.31)-(3.32), and Assumptions 3.6-3.4, one gets

$$
s_{k+1}=\widehat{a}_{k}\left(s_{k}-u_{k}\right)+u_{k}+e_{k+1} .
$$

The following results are preparatory for the stability theorem to be stated later on. They basically establish that the absolute value of the identification error grows not faster than linearly with the upper-bounding sequence $\left\{\bar{\eta}_{k}\right\}_{0}^{\infty}$ of the additive disturbances.

Assertion 3.7. If the Adaptation Algorithm 1 is used, then $\left|e_{k+1}\right| \leq \varsigma_{1} \bar{\eta}_{k}+\varsigma_{2}$, for all $k \in \mathbf{N}_{0}$, for some real constants $\varsigma_{1}(\geq \varsigma) \in \mathbf{R}^{+}$and $\varsigma_{2} \in \mathbf{R}_{0}^{+}$.

Proof. If $\left|e_{k+1}\right| \leq \varsigma \bar{\eta}_{k}$, the assertion is true with $\varsigma_{1}=\varsigma \in \mathbf{R}^{+}$and $\varsigma_{2}=0$. If $\left|e_{k+1}\right|>\varsigma \bar{\eta}_{k}$, then, from Theorem 3.2, (i) $0<\left(\left|e_{k+1}\right|-\varsigma \bar{\eta}_{k}\right) /\left(1+\gamma_{k}\left(s_{k}-u_{k}\right)^{2}\right)^{1 / 2} \leq M<\infty$, for all $k \in \mathbf{N}_{0}$. Thus

$$
\left|e_{k+1}\right| \leq s \bar{\eta}_{k}+M\left(1+\sqrt{r}_{k}\left|s_{k}-u_{k}\right|\right) \leq(s+M \sqrt{\gamma}) \bar{\eta}_{k}+M \sqrt{\gamma}\left|u_{k}\right|+M
$$

and the assertion follows with

$$
\varsigma_{1}=\varsigma+M \sqrt{\gamma}, \quad \varsigma_{2}=M(1+\sqrt{\gamma}) \operatorname{Max}_{0 \leq k<\infty}\left(\left|u_{k}\right|\right)<\infty
$$

since $\operatorname{Min}_{0 \leq k<\infty}\left(K_{k}\right)>0$.

Assertion 3.8. If the Adaptation Algorithm 2 is used and Assumption 3.4 holds, then $\left|e_{k+1}\right| \leq$ $\varsigma_{1} \bar{\eta}_{k}+\varsigma_{2}$, for all $k \in \mathbf{N}_{0}$, with

$$
\varsigma_{1}=1+\frac{2\left(\varepsilon_{1}-1\right) \varepsilon_{\mu}}{\left(1+\varepsilon_{1} \varepsilon_{\mu}\right)\left(1+\varepsilon_{\mu}\right)}, \quad \varsigma_{2}=\underset{0 \leq i<\infty}{\operatorname{Max}}\left(\left|\tilde{a}_{i}\right|\right) \underset{0 \leq i<\infty}{\operatorname{Max}}\left(\left|u_{i}\right|\right) .
$$

Proof. It follows from Assumption 3.4, (3.4) and (3.29), and the fact that $\left\{K_{k}\right\}_{0}^{\infty}$ is positively lower bounded, which yield directly

$$
\begin{aligned}
\left|e_{k+1}\right| & \leq\left|\tilde{a}_{k}\left(s_{k}-u_{k}\right)\right|+\bar{\eta}_{k} \\
& \leq\left(1+\operatorname{Max}_{0 \leq i<\infty}\left(\left|\tilde{a}_{i}\right|\right)\right) \bar{\eta}_{k}+\operatorname{Max}_{0 \leq i<\infty}\left(\left|\tilde{a}_{i}\right|\right) \operatorname{Max}_{0 \leq i<\infty}\left(\left|u_{i}\right|\right) \leq s_{1} \bar{\eta}_{k}+s_{2}, \quad \forall k \in \mathbf{N}_{0} .
\end{aligned}
$$

From Assertions 2.3 and 3.7, for the Adaptation Algorithms 1 and 2, respectively, Assumption 3.6 and (3.28c),

$$
\begin{aligned}
s_{k+1} & \leq\left|a_{k}^{*} s_{k}\right|+\left|1-a_{k}^{*}\right| r_{k}+\varsigma_{1} \bar{\eta}_{k}+\varsigma_{2} \\
& \leq \rho^{k} s_{0}+\frac{1-\rho^{k}}{1-\rho}\left(\varsigma_{1} \lambda_{1} \operatorname{Max}_{0 \leq i \leq k+1}\left(s_{k}\right)+\rho_{1} \rho_{2}+\varsigma_{1} \lambda_{2}+\varsigma_{2}\right) \\
& \leq \frac{\varsigma_{1} \lambda_{1}}{1-\rho} \operatorname{Max}_{0 \leq i \leq k+1}\left(s_{k}\right)+\frac{1}{1-\rho}\left(\rho_{1} \rho_{2}+\varsigma_{1} \lambda_{2}+s_{2}\right)+s_{0}, \quad \forall k \in \mathbf{N}_{0},
\end{aligned}
$$


for any $s_{0}>0$ and some positive finite constants $\rho, \rho_{1}$, and $\rho_{2}$ satisfying $1>\rho \geq \operatorname{Max}_{0 \leq k<\infty}\left(a_{k}^{*}\right)$, $\rho_{1} \leq 1-\operatorname{Min}_{0 \leq k<\infty}\left(a_{k}^{*}\right)<1$, and $\rho_{2} \geq \operatorname{Max}_{0 \leq k<\infty}\left(r_{j}\right)$. The last two inequalities of (3.39) follow directly by proceeding recursively with the preceding one by using $\sum_{i=0}^{k-1} \rho^{i}=\left(1-\rho^{k}\right) /(1-\rho) \leq$ $1 /(1-\rho)$ since $\rho \in(0,1)$. It is obvious that, for each $k \in \mathbf{N}_{0}$, it exists a unique $k^{\prime}(k) \in \mathbf{N}_{0}$, dependent in general on $k$, such that $k^{\prime}(k) \leq k$ such that $s_{k^{\prime}}=\operatorname{Max}_{0 \leq j \leq k^{\prime}}\left(s_{j}\right)=\operatorname{Max}_{0 \leq j \leq k}\left(s_{j}\right)$ and there is no other $j \in \mathbf{N}_{0}$ such that $k \geq j>k^{\prime}(k)$, where the same maximum is reached. A precise definition is

$$
\begin{aligned}
& k^{\prime}(k):=\operatorname{Arg}( j \in \mathbf{N}_{0}: 0 \leq j \leq k \wedge s_{k^{\prime}}=\operatorname{Max}_{0 \leq j \leq k^{\prime}(k)}\left(s_{j}\right)=\operatorname{Max}_{0 \leq j \leq k}\left(s_{j}\right), \\
&\left.\neg \exists j\left(k^{\prime}(k)<j \leq k\right) \in \mathbf{N}_{0}: s_{j}=\operatorname{Max}_{0 \leq j \leq k}\left(s_{j}\right)\right)
\end{aligned}
$$

with " $\wedge$ " and " $\neg$ " being the conjunction and negation logic symbols. The substitution of (3.40) into (3.39) yields

$$
\operatorname{Max}_{0 \leq j \leq k}\left(s_{j}\right) \leq(1-\vartheta)^{-1} C<\infty, \quad \forall k \in \mathbf{N}_{0}
$$

provided that $\varsigma_{1} \lambda_{1} /(1-\rho)<1$, which holds if and only if $0 \leq \lambda_{1}<(1-\rho) / \varsigma_{1}$ (i.e., if Assumption 3.6 holds with sufficiently small $\lambda_{1}$ ), where

$$
\vartheta:=\frac{\varsigma_{1} \lambda_{1}}{1-\rho} \quad C:=\frac{1}{1-\rho}\left(\rho_{1} \rho_{2}+\varsigma_{1} \lambda_{2}+\varsigma_{2}\right)+s_{0}
$$

with $C$ being a finite positive real, which depends on the initial conditions of the IBHE (and thus on the initial conditions of the BHE) on the available upper bound of the additive disturbances, on the reference, and on the free-design parameters of the adaptation algorithm, $\gamma_{k}$, $\varsigma>1, \xi_{0}, \xi_{1}$, and so on. The above result also holds for the Adaptation Algorithm 2 with specific constants redefined by

$$
\begin{aligned}
\rho & :=\frac{1}{1+\varepsilon_{\mu}}<1, \quad \rho_{1}^{\prime}:=\frac{\varepsilon_{1} \varepsilon_{\mu}}{1+\varepsilon_{1} \varepsilon_{\mu}}, \quad \rho_{1}:=\frac{\rho_{1}^{\prime}}{1+\varepsilon_{1} \varepsilon_{\mu}}=\frac{\varepsilon_{1} \varepsilon_{\mu}}{\left(1+\varepsilon_{1} \varepsilon_{\mu}\right)^{2}}, \\
\rho_{2} & :=\varepsilon_{1}\left(1+\varepsilon_{\mu}\right) \operatorname{Max}_{0 \leq k<\infty}\left(K_{k}^{*}\right)^{-1}<\infty,
\end{aligned}
$$

and $\xi_{1,2}$ defined in Assertion 2.3 for the Adaptation Algorithm 1 and in Assertion 3.7 for the Adaptation Algorithm 2. The boundedness of the solution of the IBHE for finite initial conditions implies that of the additive disturbances via Assumption 3.6, that of the identification error via Assertions 2.3-3.7, and that of the tracking error since the solutions of the current and the reference one are both bounded. The estimated parameters and the estimated solution are bounded from Theorems 3.2 and 3.3. Those conclusions are now summarized in the result below.

Theorem 3.9 (closed-loop stability). If Assumption 3.6 holds with sufficiently small coefficient $\lambda_{1}$, then all the signals in the closed-loop system remain bounded for all time so that the IBHE (and then the associate BHE) is globally stable if any of the Adaptation Algorithms 1 or 2 is used. 
Remark 3.10. Theorem 3.9 becomes stronger than formerly stated in the absence of additive disturbances (i.e., $\eta_{k} \equiv 0$ ) since asymptotic perfect tracking is furthermore achieved. Another result is that the identification error converges asymptotically to zero if the relative dead zone of the adaptation algorithms is removed, that is, the $\left\{\sigma_{k}\right\}_{0}^{\infty}$ is not zeroed at any sample as a result of a comparison procedure of the identification error and the additive disturbances. These results follow directly from Theorems 3.2 and 3.3 and (3.39). Note that the results may be directly generalized to the extension of using relative dead zones for adaptive algorithms by incorporating the estimation of the constants $\lambda_{1,2}$ characterizing $\left\{\bar{\eta}_{k}\right\}_{0}^{\infty}$, provided they are unknown [16].

Remark 3.11. The closeness between the IBHE and that of its reference model has been considered for feasibility reasons since, in many ecological problems, where such models are commonly of interest, the environment characteristics, which directly influence the value of the environment carrying capacity, cannot be abruptly modified even in closed environments. The employed philosophy about relative dead-zone adaptation seems to be promising to be also applied to other ecological controlled problems involving, for instance, Kolmogorov-type ecological models or models for a biochemical aquariums $[17,18]$. The obtained results seem to be promising for mathematical modeling and the use of adaptive control in other kinds of problems related to biology and ecology (see, e.g., [20-22]) and to extend the research to governing extinction conditions [10].

\section{Numerical examples}

Example 4.1. The objective is that the BHE solution $\left\{x_{k}\right\}_{0}^{\infty}$ tracks a reference sequence $\left\{x_{k}^{*}\right\}_{0}^{\infty}$ by synthesizing a control system in order that IBHE matches a reference model. Such a reference model is another IBHE defined by the appropriated sequences $\left\{\mu_{k}^{*}\right\}_{0}^{\infty}$ and $\left\{K_{k}^{*}\right\}_{0}^{\infty}$ to generate the sequence $\left\{s_{k}^{*}\right\}_{0}^{\infty}$ associated to the desired $\left\{x_{k}^{*}\right\}_{0}^{\infty}$ to be tracked by the BHE solution. The reference values are $\mu_{k}^{*}=1.55$, and $K_{k}^{*}=170$, for all $k \in \mathbf{N}_{0}$, are used for the reference model and $\mathrm{BHE}$, respectively, with the period $T=1$ (one year). It is assumed that small local variations of the carrying capacity of the habitat are allowed. The evolution data of the population of cod in the North Atlantic Sea in the period 1952-1992 are given in [4], and exhibit a quasiperiodic behavior of the intrinsic growth rate. Based on these data, the intrinsic growth rate is modeled as a sinusoid defined by $\mu_{k}=2.3+0.15 \sin (K T / 100)$, which adjusts closely to those experimental data. The average value of $K_{k}$ is 200 , what is used as a fixed uncontrolled carrying capacity for the whole set of data. The initial population of the cod is $x_{0}=500$ being identical to the reference value. Figure 1 displays the population of cod if the BHE is controlled and if it is not controlled.

The population of the controlled BHE tracks perfectly the reference sequence as in the transient as in the stationary regime for all time since the true parameterization of the BHE is known, and then the control law is not adaptive. Note, however, the uncontrolled BHE does not track the reference. Figure 2 displays the controlled carrying-capacity sequence, which leads to a perfect tracking of the reference.

Example 4.2. A numerical adaptive example is now discussed. It is assumed that the environment carrying capacity of the reference model-environment carrying capacity is a constant value of 200, and the reference intrinsic growth rate is constant of value 2 . The objective is to design the environment carrying-capacity sequence via estimation and feedback so that 


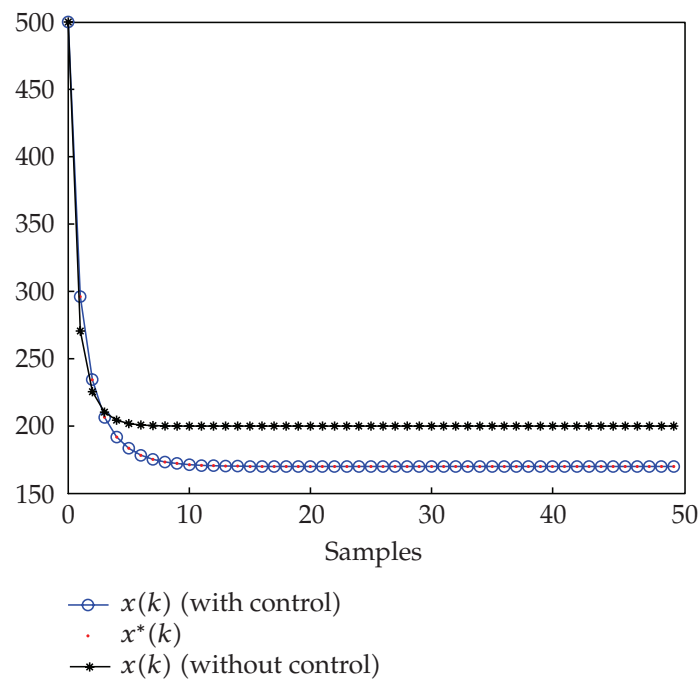

Figure 1: Evolution of the population of cod from $x_{0}=500$.

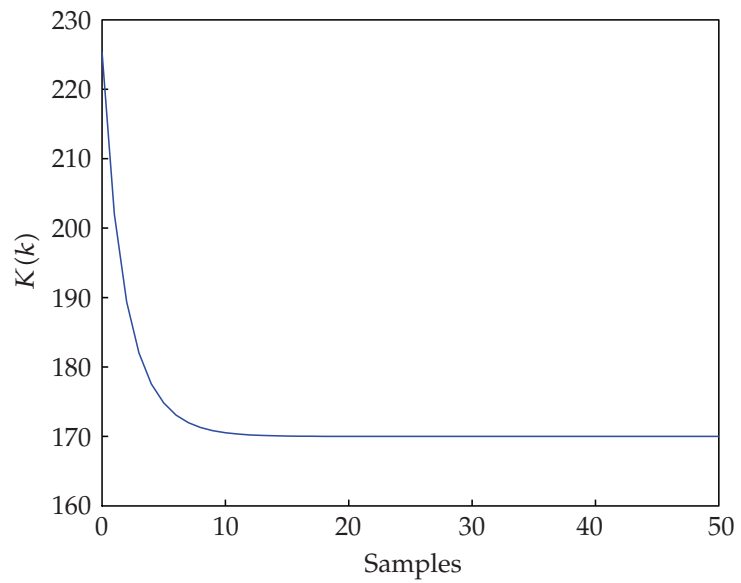

Figure 2: Evolution of the control sequence for the solution of Figure 1.

asymptotic tracking of the reference solution is achieved with positive solutions. It is assumed that no unmodeled dynamics or parametrical uncertainties are present so that a standard recursive least-squares algorithm is used for parametrical estimation of the intrinsic growth rate whose initial value is chosen and the initial value of the time-decreasing covariance gain is fixed to 1000. Figure 3 displays the reference BHE, the uncontrolled BHE, and the controlled one. It is seen that the reference BHE is asymptotically tracked, while the uncontrolled one differs from the reference. The carrying capacity of the unknown uncontrolled BHE varies from 180 to 220 according to the shape of Figure 4, and its intrinsic growth rate is constant, equal to 1.6. The carrying capacity exerting the control action is also displayed on Figure 4 with maximum and minimum admissible values being constrained by the adaptive controller to 280 and 200 , respectively. 


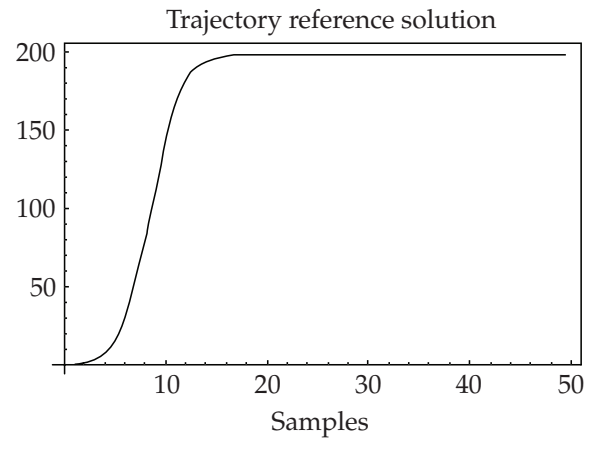

(a)

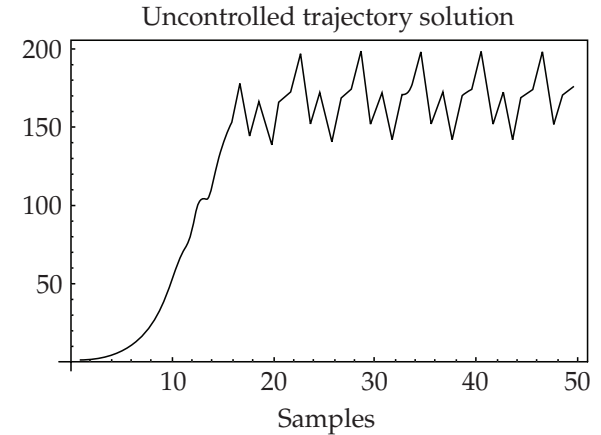

(b)

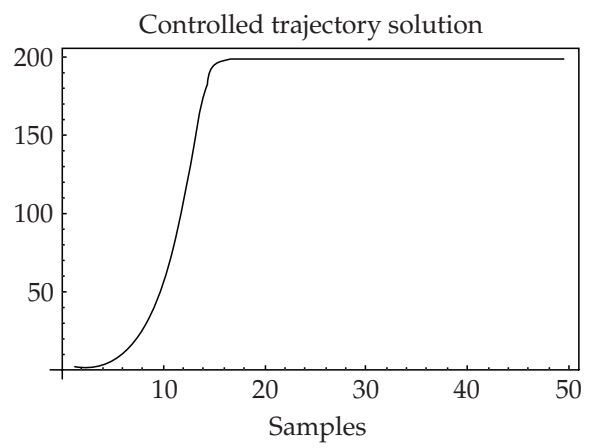

(c)

Figure 3: BHE Solutions: (a) reference, (b) uncontrolled, and (c) controlled for initial covariance gain 1000.

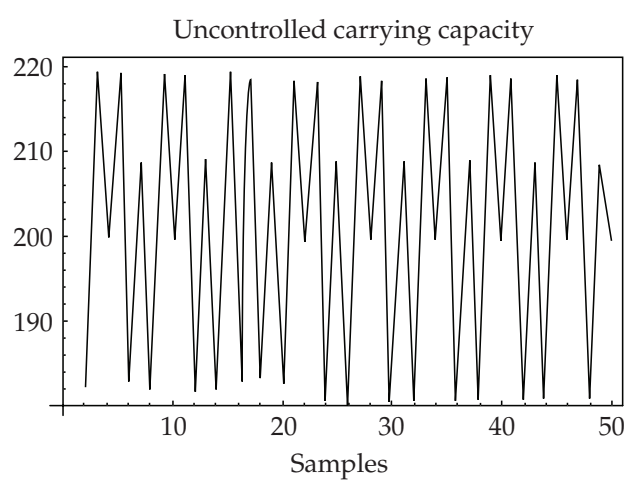

(a)

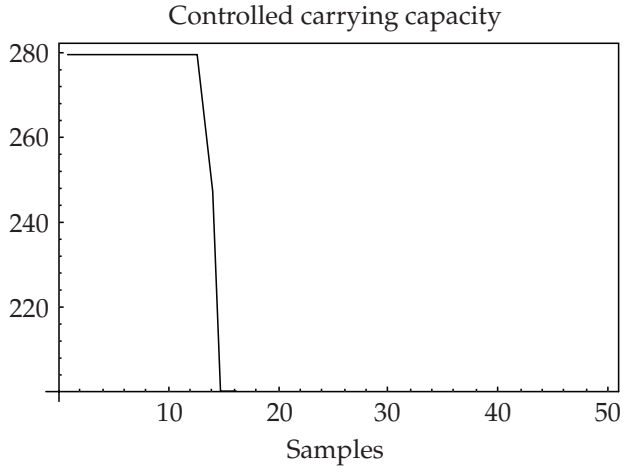

(b)

Figure 4: Environment carrying capacities: (a) uncontrolled BHE and (b) controlled.

\section{Conclusions}

This paper has considered the well-known BHE used in ecology within a control problem context where the control action on the linear IBHE is the inverse of the carrying capacity. An extended version of the standard BHE has been considered by incorporating additive 
disturbances. The overall control problem is firstly stated on the IBHE by taking advantage of its linear nature. This point of view is very feasible in close or semi-open environments where humidity, temperature, and other factors of the environment may be selected within certain margins. A reference is also defined, which describes the suited behavior for the system, the control action having an objective that the solution of the current BHE is able to perfectly track that of the reference (a). For feasibility purposes, the overall problem is stated in terms of a local variation of the inverse carrying capacity to perform the control action on the IBHE so that the reference (a) is fully tracked. This implicitly means that, in practice, the current parameters of the BHE and then those of the IBHE are locally deviated from those of its reference model. Then the method has been extended by incorporating an adaptive version for the case when the BHE parameterization is partly or fully unknown. The use of a relative adaptation dead zone freezes the adaptation when the identification error is sufficiently small according to an available absolute upper bound of the disturbances sequence so that the algorithm is proven to prevent potential instability caused by the presence of such additive disturbances. The second one incorporates to the estimation dead zone and estimates projection procedure by using a priori knowledge on the parameters of the BHE and the second algorithm, which have been presented. It has been proven that both adaptation algorithms and associate control law stabilize the current, provided that the absolute value of the additive disturbances grows not faster than linearly with the maximum of the solution with a sufficiently small slope. Some related numerical examples have been discussed to corroborate the theoretical results. The potential generalization to the general case, where the parameterizations of the current BHE and its reference one to be tracked are not close to each other, is direct although it may be not feasible in some practical cases, since the adaptation algorithms and the main stability results are formulated in a general way.

\section{Acknowledgments}

The authors are very grateful to the Spanish Ministry of Education for its partial support of this work through Project DPI 2006-00174. They are also grateful to the referees for their useful comments.

\section{References}

[1] N. J. Barrowman, R. A. Myers, R. Hilborn, D. G. Kehler, and C. A. Field, "The variability among populations of coho salmon in the maximum productive rate and depensation," Ecological Applications, vol. 13, no. 3, pp. 784-793, 2003.

[2] A. L. Jensen, "Harvest reference points for the Beverton and Holt dynamic pool model," Fisheries Research, vol. 47, no. 1, pp. 93-96, 2000.

[3] M. Holden, "Beverton and Holt revisited," Fisheries Research, vol. 24, no. 1, pp. 3-8, 1995.

[4] G. Stefansson, "Fish 480 (stockrec) spawning stock, recruitment and production," Course at the Department of Biology of the University of Iceland using data of the Marine Research Institute of Reykjavik, Iceland, November 2005.

[5] R. J. H. Beverton and S. J. Holt, "On the dynamics of exploited fish populations," Fisheries Investment, vol. 19, p. 1, 1957.

[6] C. Hui, "Carrying capacity, population equilibrium, and environment's maximal load," Ecological Modelling, vol. 192, no. 1-2, pp. 317-320, 2006.

[7] M. A. McCarthy, "The Allee effect, finding mates and theoretical models," Ecological Modelling, vol. 103, no. 1, pp. 99-102, 1997. 
[8] M. De la Sen, "Parameter dependent Lyapunov functions for robust stability of time-varying linear systems under point delays," Applied Mathematics and Computation, vol. 179, no. 2, pp. 612-621, 2006.

[9] S. Stević, "A short proof of the Cushing-Henson conjecture," Discrete Dynamics in Nature and Society, vol. 2006, Article ID 37264, 5 pages, 2006.

[10] M. De la Sen, "The environment carrying capacity is not independent of the intrinsic growth rate for subcritical spawning stock biomass in the Beverton-Holt equation," Ecological Modelling, vol. 204, no. 1-2, pp. 272-273, 2007.

[11] M. De la Sen, “On impulsive time-varying systems with unbounded time-varying point delays: stability and compactness of the relevant operators mapping the input space into the state and output spaces," The Rocky Mountain Journal of Mathematics, vol. 37, no. 1, pp. 79-129, 2007.

[12] M. De La Sen and S. Alonso, "Adaptive control of time-invariant systems with discrete delays subject to multiestimation," Discrete Dynamics in Nature and Society, vol. 2006, Article ID 41973, 27 pages, 2006.

[13] S. Alonso-Quesada and M. De la Sen, "Robust adaptive control of discrete nominally stabilizable plants," Applied Mathematics and Computation, vol. 150, no. 2, pp. 555-583, 2004.

[14] M. De la Sen and N. Luo, "On the uniform exponential stability of a wide class of linear time-delay systems," Journal of Mathematical Analysis and Applications, vol. 289, no. 2, pp. 456-476, 2004.

[15] M. De la Sen, "Robust stable pole-placement adaptive control of linear systems with multiestimation," Applied Mathematics and Computation, vol. 172, no. 2, pp. 1145-1174, 2006.

[16] G. Feng, "Analysis of a new algorithm for continuous-time robust adaptive control," IEEE Transactions on Automatic Control, vol. 44, no. 9, pp. 1764-1768, 1999.

[17] Z. Huang, S. Chen, and Y. Xia, "Incorporate intelligence into an ecological system: an adaptive fuzzy control approach," Applied Mathematics and Computation, vol. 177, no. 1, pp. 243-250, 2006.

[18] R. Fazio and A. Jannelli, "Mathematical and numerical modeling for a bio-chemical aquarium," Applied Mathematics and Computation, vol. 174, no. 2, pp. 1370-1383, 2006.

[19] F. Chen and C. Shi, "Global attractivity in an almost periodic multi-species nonlinear ecological model," Applied Mathematics and Computation, vol. 180, no. 1, pp. 376-392, 2006.

[20] X. Ding and H. Su, "Dynamics of a discretization physiological control system," Discrete Dynamics in Nature and Society, vol. 2007, Article ID 51406, 16 pages, 2007.

[21] N. Hritonenko, A. Rodkina, and Y. Yatsenko, "Stability analysis of stochastic Ricker population model," Discrete Dynamics in Nature and Society, vol. 2006, Article ID 64590, 13 pages, 2006.

[22] N. Kamel, A. Mousavi, and A. Samraj, "Whitening of background brain activity via parametric modeling," Discrete Dynamics in Nature and Society, vol. 2007, Article ID 48720, 11 pages, 2007. 


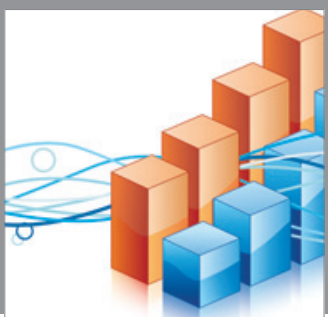

Advances in

Operations Research

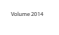

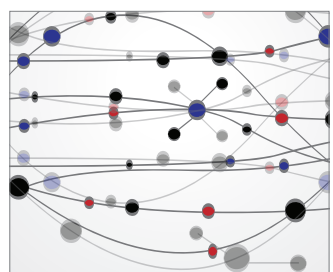

\section{The Scientific} World Journal
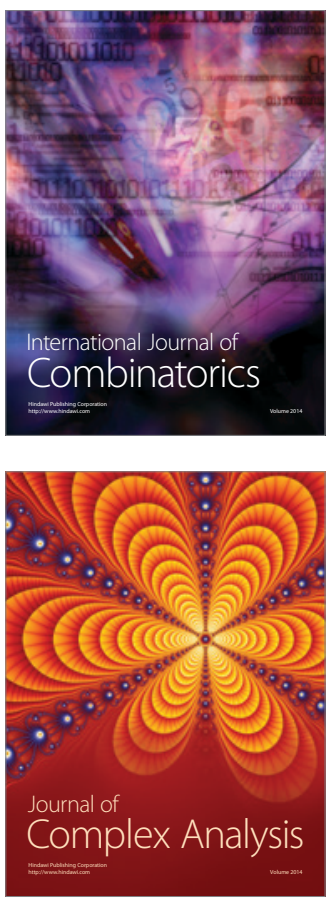

International Journal of

Mathematics and

Mathematical

Sciences


Journal of

Applied Mathematics
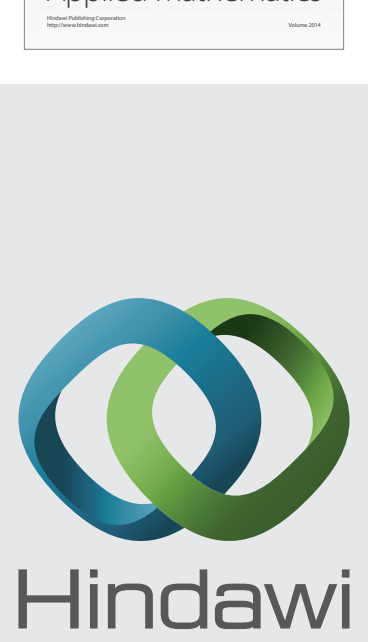

Submit your manuscripts at http://www.hindawi.com
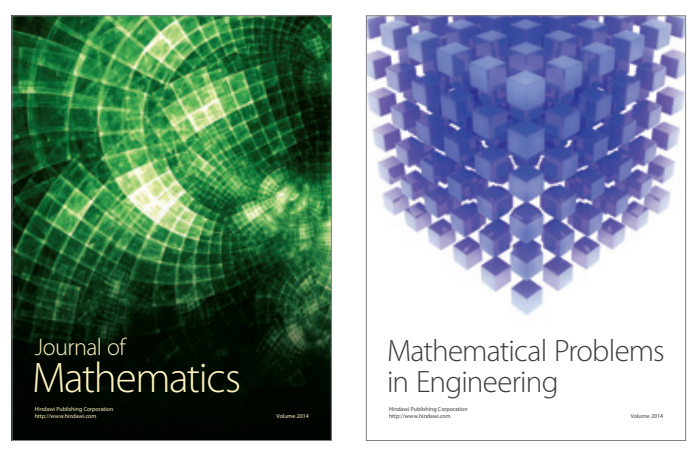

Mathematical Problems in Engineering
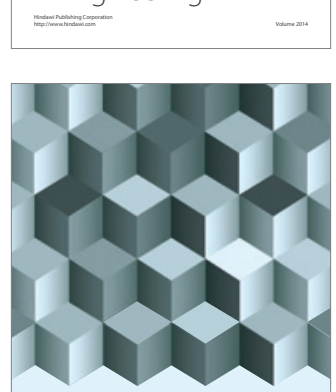

Journal of

Function Spaces


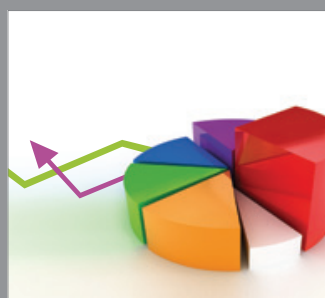

ournal of

Probability and Statistics

Promensencen
